\title{
Störungsspezifische oder verhaltensanalytisch basierte Psychotherapie?
}

\author{
Michael Linden \\ Forschungsgruppe Psychosomatische Rehabilitation, Charité Universitätsmedizin Berlin, Berlin, Deutschland
}

Als die Krankenkassen für die Bezahlung von Psychotherapie aufkommen sollten, haben sie gefordert, dass die therapeutische Wirksamkeit von Psychotherapieverfahren «störungsspezifisch» nachgewiesen wird, beispielsweise unter Bezug auf ICD-Diagnosen (ICD = International Statistical Classification of Diseases and Related Health Problems). Entsprechend prüft auch der Wissenschaftliche Beirat Psychotherapie, ob Wirksamkeitsnachweise bei eindeutig diagnostizierten Krankheitsfällen vorliegen, in Abgrenzung zu allgemeinen Lebensproblemen.

Wissenschaftstheoretisch gilt, dass Diagnosen hypothetische Konstrukte sind, die nach mehr oder weniger konsentierten Regeln klinische Problemstellungen in einem Wort zusammenfassen und damit signalisieren, dass ein Krankheitsfall im sozialrechtlichen Sinne vorliegt. Eine ICD-Diagnose rechtfertigt eine Therapie oder sonstige soziale Leistungen und ist damit für die Sozialkassen die entscheidende Voraussetzung für eine Leistungserbringung. Die ICD oder das DSM geben hierfür Codes vor, die eine Gesundheitsstatistik ermöglichen, und heißen daher nicht von ungefähr «Internationale statistische Klassifikation der Krankheiten» bzw. «Diagnostisches und statistisches Manual psychischer Störungen». Sie sind keine Lehrbücher und geben in vielen Bereichen auch nur globale Oberkategorien vor, sodass es deutlich mehr Krankheiten als ICD-Codes gibt.

Eine Diagnose nach der ICD ist zu unterscheiden von der Beschreibung des Krankheitsfalls. Eine Diagnose kann aufgrund ihrer Kondensiertheit auch nicht therapieleitend sein, wenngleich dies unter dem Stichwort der «störungsspezifischen Therapie» gelegentlich nahegelegt wird. So sagt beispielsweise die Diagnose eines Herzinfarkts oder einer bipolaren Störung wenig darüber aus, wie therapeutisch vorzugehen ist. Bei einigen Formen von Herzinfarkten sind keine weiteren therapeutischen Interventionen erforderlich, andere bedürfen einer Behandlung auf einer Intensivstation. Die Behandlung einer bipolaren Störung hängt nicht nur von der Diagnose, sondern von der Rezidivfrequenz und dem aktuellen Befund $\mathrm{ab}$ und kann dementsprechend sehr unterschiedlich ausfallen.
Somit orientiert sich die Therapie nicht primär an der «Diagnose», sondern an der «Diagnostik», d.h. der Feststellung des aktuellen Krankheitsstatus.

Einen konzeptionellen Rahmen hierfür bietet beispielsweise die Internationale Klassifikation der Funktionsfähigkeit, Behinderung und Gesundheit (ICF), die vor einigen Jahren von der Weltgesundheitsorganisation neu herausgegeben wurde (wie auch die ICD). Sie unterscheidet zwischen Körperstrukturen, Körperfunktionen (= Symptomen), Fähigkeiten, Kontext und Teilhabe und ist damit eine Operationalisierung des klassischen «ganzheitlichen» oder «bio-psycho-sozialen» Krankheitsverständnisses.

Diese ICF-Perspektive ist gerade für die Psychotherapie von besonderer Bedeutung. Seit jeher haben sich psychotherapeutische Prozesse weniger an Diagnosen als vielmehr an Befunden und vor allem an Krankheitsmodellen orientiert - also beispielsweise in der psychodynamischen Therapie an Komplexen, in der klientenzentrierten Psychotherapie an der Diskrepanz zwischen Ideal- und Real-Selbst oder in der Verhaltenstherapie an der Verhaltensanalyse. Auch üblicherweise als «störungsspezifisch» bezeichnete Psychotherapiemethoden sind keineswegs störungsspezifisch, sondern ihre Techniken werden in der Regel befundabhängig (anstatt primär abhängig von der jeweiligen ICD-Diagnose) bei unterschiedlichsten Erkrankungen eingesetzt, seien es das Skill-Training gemäß der dialektisch-behavioralen Therapie oder ein Selbstsicherheitstraining bzw. die Modifikation dysfunktionaler Kognitionen in der Verhaltenstherapie. Gleichzeitig gibt es auch bezüglich der jeweiligen «Störung», z.B. Borderline, Depression, Trauma- oder Zwangsstörung, diverse alternative Therapieansätze und Therapievarianten.

Die Übersetzung des ICF-Konzepts in die psychotherapeutische Anwendung erfolgt in der Verhaltenstherapie über die Verhaltensanalyse. So können sich beispielsweise hinter der Diagnose «Zwangserkrankung» (F 42.1) unter verhaltensanalytischer Perspektive sehr unterschiedliche Störungen verbergen, etwa ein stimulusbedingtes Angst-Vermeidungsverhalten, eine Ekelkonditionierung, magisches Denken, Neutralisierungsprozesse, Probleme

\section{KARGER}

() 2018 S. Karger GmbH, Freiburg

Fax +497614520714 
mit dem Kurzzeitgedächtnis, Unvollständigkeitserleben oder eine Impulskontrollstörung. Jeder dieser psychologischen Prozesse ist therapeutisch unterschiedlich anzugehen und je nach Fall auch von unterschiedlicher Bedeutung. Dies wird in der Verhaltenstherapie als «Störungsmodell» bezeichnet und ist die Grundlage für die Planung und Durchführung einer Therapie. In nahezu allen Anträgen auf Psychotherapie wird dementsprechend von den Therapeuten ausgeführt, dass die Erarbeitung und Vermittlung eines Störungsmodells ein wichtiges Therapieelement sei.

Im Kern handelt es sich also um ein individualisiertes Konzept von kranken Menschen, das auch in der aktuellen Forschung unter den Stichwörtern "personalisierte Medizin», «transdiagnostische Behandlungsansätze» oder «Präzisionsmedizin» diskutiert wird. Auch die Krankenkassen kennen den Unterschied zwischen einer Diagnose und einem Störungsmodell. Sie bestehen einerseits darauf, dass eine Diagnose gestellt wird, da sie nicht für die Unterstützung bei der Lösung allgemeiner Lebensprobleme bezahlen wollen. Andererseits verlangen sie dann aber auch explizit, dass anhand eines «Störungsmodells» (siehe z.B. Punkt 2 der Kurzbegründung des Gutachters für die Krankenkasse auf Formblatt PTV5) die Zweckmäßigkeit einer Behandlungsplanung dargelegt wird.

In neuerer Zeit wurde immer wieder eine primär störungsspezifische Psychotherapie propagiert und sogar als Ausbildungsrichtschnur gefordert. Diese Sichtweise greift zu kurz, da sie der Vielfalt der Störungen und der Individualität der Patienten nicht gerecht wird. Sie kann sogar zu Fehlbehandlungen führen und ist sicher keine Ausbildungsgrundlage. Stattdessen hat der personalisierte Ansatz der Verhaltenstherapie durch die Einführung der ICF zusätzlich an Aktualität gewonnen. Die Verhaltensanalyse muss das zentrale Element im Fallverständnis, in der Behandlungsplanung und in der Therapieevaluation bleiben. Und daher muss sie auch die gebotene Aufmerksamkeit in der Ausbildung von Verhaltenstherapeuten erhalten.

\section{Disclosure Statement}

Der Autor ist verhaltenstherapeutischer Behandler, Supervisor und Dozent. 